\title{
Lepra mit besonderer Berücksichtigung der Uebertragung durch Heredität oder Contagion.
}

\author{
Referat-Vortrag, gehalten in der XVI. Section des X. internationalen \\ medicinischen Congresses zu Berlin. - August 1890. \\ Von
}

Dr. Ed. Arning - Hamburg.

Meine Herren!

Alle, die Sie hier versammelt sind, um über gewichtige Fragen der Volkserkrankungen Thre Meinungen auszutauschen, sind sich dessen bewusst, dass es im eigentlichen Sinne schwebende Fragen sind, die in den Discussionen belenchtet werden sollen. Kaum eine dér vorgebrachten Fragen dürfte voll beantwortet und erledigt werden, kaum eine aber andererseits durch Ihre massgebenden Meinungsäusserungen nicht dor ondgiltigen Lösung nälıer gebracht worden. So steht es auch um die Leprafrage, die ich einzuführen die lihre babe, und bosonders um. den Kernpunkt der ganzen Lepraforschung: ob Contagion, ob Heredität die wesentlichere Rolle bei der Verbreitung dor Krankheit spielt.

Sie worden nun nicht von mir erwarten, meine Herren, dass ich bei Betrachtung dieser Frage, die so alt ist, wie unsere früheste systematische Kunde von den Erkrankungen des Menschengeschlechtes, wieder ab ovo beginne. Nur die allerneuesten Phasen der Lepraforschung möchte ich in den Kreis der Betrachtung ziehen, und auch aus der modernen Hochfluth der Lepraliteratur nur dasjenige herausgreifen, was zur Klärung der uns in dieser Section beschäftigenden Fragen direct beitragen kann.

Um zunächst festen Grund zu fassen für unsero Discussion, so stehen wir wohl Alle auf dem standpunkte, dass die Lepra 
eine Infectionskrankheit ist, und dass wir die Träger dieser Infection in den durch die classischen Untersuchungen von Hansen und Neisser bekannt gewordenen Leprabacillen zu suchen haben. Ich vermag mir keine Förderung unserer Kenntniss der Lepra-Krankheit vorzustellen, welche nicht von dieser Grundlage ausgeht. Andererseits müssen wir uns aber auch sagen, dass diese damals als Offenbarung begrüsste Entdeckung vorläufig eben nur diesen festen Boden geschaffen hat. Die erhofften, sich unmittelbar weiter daraus ergebenden Folgerungen sind ausgeblieben. Zwar wissen wir, dass wo immer Lepra existirt, da anch die Leprabacillen vorbanden sind; wir wissen, dass sie bei gewissen Formen der Lepra in ungehenren Mengen die Haut und die Eingeweide durchsetzen, und dass wir sie andererseits auch bei den reinen Formen der sogenannten Lepra anaesthetica in den Nervenstämmen finden können. Wir wissen aber nicht, was für Bodingungen zur Aufnahme des Bacillus vorhanden sein müssen, wir wissen nicht wie er in den Körper eindringt und wie er sich dort vermehrt, wir wissen schliesslich noch nichts über die Lebensbedingungen, Wachsthumsformen und Stoffwechselproducte dieser Krankheitserreger.

So müssen wir uns vorläufig noch mehr an klinische und epidemographische Facta und Erwägungen halten, und ich will nun gleich zu dem ersten Thema ühergehen: Ist die Lepraübertragung durch Heredität wahrscheinlich oder erwiesen?

Den Typus der hereditär vermittelten Krankheiten bildet die Syphilis. Eine Ungehung des Herelitätsbegriffes ist eigentlich schon die für die T'uberculose geforderte erblich übertragene Disposition. Bei der Syphilis finden wir alle Grade der directen Uebertragung vou der bereits in ntero an der ererbten Krankheit gestorbenen Frucht, durch die mit manifesten Symptomen geborenen oder bald nach der Geburt dieselben entwickelnden Kinder, bis zu den Fällen, wo die Krankheit der Eltein sich nur in mehr oder weniger allgemeinen Ernährungsstörungen der Kinder äussert.

Nichts von allen dem bei der Lepra. Dd gibt es keine fötale Erkrankung, kaum eine infantile. Nur ganz vereinzelte Fälle sind berichtet worden, wo in dem Verlaufe der ersten beiden Lebensjahre die Lepra sich entwickelte, und auch dann tritt sie ganz so auf wie bei den Erwachsenen, sie hat nicht wie die 
Syphilis bestimmte, typische Erscheinungsformen, wodurch man bei dieser dis ererbte Krankheit von der erworbenen unterscheiden kann.

Wir werden also aus dem klinischen Bilde der Lepra bei Kindern keine Beweise oder auch nur Stützen für die Theorie der Herodität der Krankheit ableiten können. Aber auch anderweitig spricht die klinische Beobachtung dagegen, dass bei grossartiger, endemischer Verbreitung der Krankheit die Heredität als wesentlicher Factor in Betracht kommt. Bei Mädchen, die vor der Pubertätszeit leprös erkranken, findet sich fast immer ein Zurückbleiben in sexueller Beziehung hinter ihren gesunden Altersgenossen. Sie entwickelı sich spät und unvollkommen, und besonders die ovariellen Functionen sind beeinträchtigt. Das mag zuweilen auf einer leprösen Ovariitis beruhen, wie ich sie bei ,einem an Lepra verstorbenen jungen Mädchen habo nachweisen können. Im Stroma des Ovariums fand sich eine entzündliche Infiltration, als derou Ursache die weit durch das Organ vertheilten Leprabacillen anzusehen war. Kommt die Lepra bei bereits menstruirten Frauen zum Ausbruch, so ist auch hier eine Störung der menstruelleu Functiouen häufig zu bemerken, und bei chronischen Fällen tritt die Menopause oft abnorm frühzeitig ein. Was von dem Weibe gilt, gilt in noch viel höherem Masse vom Manne. Hier wissen wir schon lange, dass bei allgomeiner Leprak die Hoden fast regelmässig befallen werden. Im Gegensatze zu älteren Berichten über eine Satyriasis leprosa ist noueren Lrfahrungen nach die Potentia virilis bei Leprösen fast immer herabgesetzt, und erlischt häufig vollständig schon nach wenigen Jahren der manifesten Erkrankung. Es scheint mir das ein gewaltiger Unterschied der Lepra von Syphilis und Tuberculose zu sein und es will mir immer sonderbar vorkommen, wie man eine Krankheit, die schon so frühzeitig und gründlich die Geschlechtsfunctionen stört, wesentlich durch Vererbung verbreitet wissen will.

Damit soll natürlich nicht gesagt sein, dass bei Lopra eines oder beider Eltern etwa vor der Krankheit oder auch nach Ausbruch derselben noch geborene Kinder nicht leichter und häufiger erkranken als andere. Im Gegentheil, ich stehe voll und ganz auf dem Standpunkte, dass die Lepra mit Vorliebo als Familionkrankheit auftritt, aber soweit ich sehen kam, hindert uns nichts, 
eine derartige familiär begrenzte Ausbreitung der Erkrankung einer frühzeitigen Infection unter dem Einflusse des leprösen Privatklimas der Familie zuzuschreiben. Das ist eine wahrlich ungezwungenere Annahme als die einer supponirten Heredität mit undefinirbar begrenzter Latenzperiode.

Nun kommt hinzu, dass bei solchen leprösen Familienerkrankungen es sich in durchaus nicht seltenen Fällen nachweisen lässt, dass durch ein Kind die Lepra in die Familie hineingebracht wurde, und dass erst die Kinder, d ann die Eltern erkranken. Weiterhin gehört es zu den ganz gewöhnlichen Erscheinungen in Lepraländern, dass bei Aussatz eines oder beider Eltern ein Theil der Kinder ganz gesund, ein anderer erkrankt ist, und zwar ohne irgend eine chronologische Ordnung in der Reihenfolge der Erkrankungen.

Wesentlich zur Entscheidung der Frage würde es beitragen, wenn es gelänge, einen Vorschlag durchzuführen, den ich der hawaiischen Regierung gemacht habe, und der dahin geht, noch gesunde Kinder aus leprösen Familien zu entfernen, wenn diese sich freiwillig in abgelegener Gegend segregirt haben; und auch in anderen Ländern würde ich zu solchen Massregeln rathen, wenn eine Zwangssegregation der Erkrankten selber nicht durchführbar erscheint. Bliebe ein grosser Procentsatz solchergestalt entfernter Kinder gesnnd, so würde das ein wichtiges Argument gegen die Hereditätstheorie darstellen.

Solange unsere Kenntniss des Aussatzes wesentlich auf den Lehren der älteren norwegischen Forscber beruhte, war es natürlich, dass die Lehre der Heredität prävaliren konnte. Fielen doch diese Forschungen in eine Zeit, wo die Sätze der Uebertragung der Syphilis hereditaria anfingen formulirt zu werden, und wo die HumoralPathologie mit ihren abstracten Raisonnements noch wesentlich die Geister beherrschte. Dabei entstand diese Lehre auf einem geographischen Gebiete, wo seit fünf Jahrhunderten die Lepra endomisch war und wo dio Bevölkerungsgruppen sesshaft und ohne vielen gegenseitigen Verkehr lebten. In mehr weniger tellurisch abgeschlossenen Familiengruppen pflanzte sich der Aussatz fort.

Unter solchen Umständen ist es gar nicht zu verwundern, dass die Lehre von der hereditären Uebertragung der Lepra entstand und feste Warzeln fasste. Mir will es aber immer 
scheinen, dass die Hereditätslehre der Lepra ein Erbtheil einer vorübergegangenen Aera der Pathologie ist, welches wir zunächst von uns weisen sollten, um klar und unbefangen zu sehen. Meiner Ueberzengung nach wäre diese Theorie heutzutage nie und nimmer entstanden. Ganz abgesehen davon, dass wil jetzt den Infectionsträger der Lepra kennen, haben allgemeiner Verkehr, die rasche Verquickung verschiedenartigster Nenschenrassen, und eine gewisse übersichtliche Kenntniss der hygienischen Zustände aller Theile der Erde Bedingungen geschaffen, welche uns hindrängen zu der Behauptung: die grosse und rapide Verbreitung der Lepra an solchen Stätten, die nicht alte endemische Sitzo der Krankheit darstellen, ist durch die Annahme einer ausschliesslichen oder auch nur wesentlichen Uebertragung auf' dem Vererbungswege nicht erklärbar.

Aber wir müssen auch Detailfälle gegen die Hereditätslehre zu Felde führen, und das ist bei dem eminent chronischen Verlanfe der Lepra gar nicht leicht. Man wird aus der Unsumme ron gesammeltem Material als beweiskräftig nur solche Fälle heraussuchen, wo eine anch nur ererbte Disposition, geschweige denn eine directo Vererbung ausgeschlossen ist, und wo zwischen dem Eintritt in die Zone der Infectionsmöglichkeit und der ersten Offenbarung der Lrkrankung keine allzu lange Zeit verstrichen ist.

In dieser Beziehung scheint mir folgender Fall ron einwandsfreier Bedoutung: Fine Dame in den Zwanziger Jahren kommt von einer leprafreien Gegend der Vereinigten Staaten nach Honolulu. Weder sie noch ihre Eltern oder sonstige Verwandten haben jemals in Beziehung zu Leprösen gestanden. Drei Monate nach ibrem Eintreffen in Honolulu bemerkt sie auf dem Rücken des linken Vorderarmes eine kleine rothe, leicht erhöhte Stelle, die sich langsam vergrössert und aller, zunächt allerdings sehr milder Behandlung trotat. Nach einem Jahre bemerkt die Patientin, dass die Stelle anästhetisch ist, hat aber weiter keine Beschwerden davon. Nach Verlauf von 2 Jahren findet sich eine circa thalergrosse in der Mitte leicht eingesunkene Papelgruppe, welche einem Lupus zwar ähnelt, sich aber durch ihre absolute Anästhesie sehr markant davon unterscheidet. Die Anästhesie dehnte sich bereits über den Rand der afficirten Stelle auf die umgebende 
anscheinend noch gesunde Hant aus. Unter Umständen schwoll die erkrankte Hantpartie urticariaartig an und vage, dumpfe Schmerzen zogen sich nach dem Ellbogen hin. Ich konnte die ganze Stelle chne Scbmerzgefübl entfernen. Mikroskopisch fanden sich in dem Granulationsgewebe Gruppen von Leprabacillen. Mir scheint es unzweifelhaft, dass es sich in diesem Falle um eine primäre Localisation des Lepra-Virus in der Haut gehandelt bat, und dass wir unter den geschilderten Umständen auch klimatische und tellurische Einflüsse ausschalten können. In mancher Beziebung noch massgebender als dieser Fall sind der Dubliner Fall von Hawtrey Benson ${ }^{1}$ ) und der Baltimorer Fall von Atkinson ${ }^{2}$ ); beide wohl genügend durch Leloir's schönes Leprawerk bekannt. Ich resumire nur kurz das beiden Fällen gemeinsame: In einem leprafreien Lande wird eine Person, die dieses Land nie verlassen hat und erblich unbelastet ist, von dem Aussatze ergriffen, nachdem 'sie im Verkehre mit einer anderen Person gestanden hat, welche ansserhalb dieses Landes leprös erkrankt war.

Das sind doch beinahe classische Beweise für die Uebertragbarkeit der Krankheit durch Contagion. Weniger absolut beweiskräftig, aber immerhin nicht ohne eine gewisse Tragweite, sind solche Fälle, wie sie Heidensta $\mathrm{m} \mathrm{m}^{3}$ ) kürzlich aus Cypern berichtet, und wie ich sie habe auf den hawaiischen Inseln beobachten können: Es kommt ein Leprakranker in einen District, der bisher frei von Lepra war, und im Verlanfe weniger Jahre, also viel schneller, als durch eine Verbreitung auf hereditärem Wege möglich, erkranken eine ganze Reihe von Personen, und alle diese Erkrankungen lassen sich concentrisch zurückführen auf den ersten eingeschleppten Fall.

Schliesslich möchte ich gegen die Hereditätslehre noch einige nevere Erfahrungen aus solchen Gegenden anführen, wo es neben einer durchseuchten Eingeborenen- und Mischlings-Bevölkerung eine grosse Classe von weissen Eingewanderten gibt, die als hereditär unbelastet gelten können.

So führt Blank in einem Berichte über die Lepra in Louisiana an, dass von seinen in New-Orleans gezählten 42 Fällen in

1) Dublin Medic. Journal, June 1877 .

2) Transactions of the Americ. Dermatolog. Association 1881. p. 50 .

$\left.{ }^{3}\right)$ Brit, Med, Journ. 12. April 1890. 
30 Procenten sich nachweisen liess, dass ein oder beide Eltern Europäer waren. Von diesen Kranken waren sieben in Deutschland und je einer in Oesterreich, Frankreich und Italien geboren.

Für Hawaii habe ich folgende Berechnung gemacht:

Nach dem Census von 1884 stellt sich die Zahl der eingeborenen Hawaiier und Mischlinge anf 44.232, die Zahl der Fremden ohne Chinesen und Japaner auf 17.935. Rechnet man nun gering die Zahll der loprösen Hawaiier auf 1500, so haben wir für die eingeborene Bevölkerung das schreckliche Verhältniss von einem Leprösen auf 30 Gesunde.

Unter den 17.935 Weissen kenne ich 35 Leprafälle, wobei ich natürlich nur solche zähle, die um die Zeit der Zäblung oder bald darauf erkrankt warew, und nicht die vielen bereits an Lepra gestorbenen Weissen. Auch sind alle Halbweissen aus dieser Tiste ausgeschlossen. Wir bekommen damit ein Verhältniss von ein em Leprakranken auf 514 Gesunde einer Bevölkerungsclasse, wo Heredität kaum in Frage kommt.

Schliesslich möchte ich noch kurz anf dio Resultate von Il an seu's Roise zu den nach Nordamerika ausgewanderten leprösen Norwegern linweisen. Von 160 Finwanderern fand Hansen nur noch 17 am Tueben. Von den Nachkommen dieser Norweger war kein einziger der Lepra anheimgefallen.

Dass der Aussatz von klimatischen und tellurischen Bedingungen unabhängig ist, branche ich nicht des Weiteren auszuführen. Wir müssen immer und immer wieder urgiren, dass die Lepra nur durth den leprösen Menschen versehleppt und verbreitet wird. Sie ist als exclusive Krankheit des Menschen anzusehen, jedenfalls nicht wie die Tuberculose eine Erkrankung der den Menschen umgebenden Hausthiere oder allgemein bekannter Thiergattungen. Unter besonders günstigen Umständen ist die Erkrankung höchst wahrscheinlich von Mensch zu Mensch direct ïbertragbar; eine indirecte Uebertragung durch Vermittlung der den Menschen umgebenden Medien, eventuell auch durch Nahrungsmittel ist sehr wohl denkbar; was aber speciell die letzteren angeht, durchans nicht erwiesen. Besonders erscheint mir die von Jonathan Hutchinson nelerdings wieder aufgenommene Fischtheorie in ihrer Exclusivität gegen andere Infectionsmodalitäten unhaltbar. 
Einen anderen gelegentlich der hawaiischen Lepra-Epidemie viel besprochenen, von dem verstorbenen Dr. Hillebrand besonders hervorgehobenen Punkt müssen wir näher betrachten: Ist die Lepra dort durch die allgemeine Schutzpockenimpfung verbreitet worden?

Es kann kein Zweifel über die Syuchronicität der Verbreitung der Lepra und der Einführung der Impfung auf den hawaiischen Inseln bestehen, wohl aber ist manche Fehlerquelle offen, wenu man die beiden Facta in einen engen Causalnexus bringen will.

Die ersten authentischen Leprafälle konnte ich aus dem Jahre 1830 ermitteln; die furchtbare Verbreitung über die ganze Inselgruppe trat aber erst 25 Jahre später ein, zu einer Zeit, wo eine schwere Blatternepidemie den Anlass zu einer sehr allgemeinen und meist von Laien sehr sorglos ausgeführten Vaccination gab. Diese Thatsache ist von Hillebland und Anderen als Argument für die Verbreitung des Aussatzes durch Vaccination angenommen worden. Wenn wir nun auch dieses historische Factum der Synchronicität nicht übersehen dürfen, so können wir doch auch eine andere Erklärung dafür geben. Bedenken wir, dass es bereits 1830 wohlausgebildete Leprafälle gab, so müssen wir wenigstens einige Jahre zurück die Einschleppung der Krankheit datiren. Dann haben wir in den nächsten Jahren eine langsame Verbreitung um die ersten Centren herum, und würden uns das anscheinend plötzliche Umsichgreifon im Anfange der Fünfziger Jahre dadurch plausibel machen können, dass die Lepra, wenn auch nicht congenital und hereditär, doch immerhin eine Familienkrankheit ist, und dass in der Zeit von der Einschleppung an bis zu dem in Frage stehenden Termine gerade eine neue Generation aufgewachsen war, welche nun in eigenen Haushälten von ibrem Leprafamilienfocus aus die Krankheit rasch diffundirte. Weiterhin muss man den enormen Einfluss auf die Eingeborenen nicht vergessen, welchen gerade in dieser Periode zwischen den Dreissiger und Fünfziger Jahren die Einführung der Civilisation und das Hereinströmen einer grossen mongolischen und kaukasischen Bevölkerung ausüben musste.

Ich lege einer anderen Beobachtung in Bezug auf den Zusammenhang von Lepra und Vaccination eigentlich mebr 
Ge wicht bei. Diese stammt ans einer viel späteren Periode, in welcher keine concomitirenden Factoren, wie die oben angeführten, im Spiele sind.

Alaf ausgezeichnete. Antoritut lin, leider sind statistische Angaben nicht beschaffbar, kann ich behaupten, dass eine sehr auffällige locale Häufung von neuen Leprafällen in den Jahren 1871-1872 an dem Orte Lahaina auf Mani sich bemerkbar machte, circa 1 Jahr nach ausserordentlich sorglos dort ausgeführter Arm zu Arm Taccination. Ungefähr 50-60 Fälle zeigten sich plotzlich in diesem Districte, der bisher verhältnissmässig frei von Lepra gewesen war.

Einige wenjge directe Versucho ${ }^{1}$ ), die ich zur Klärung dieser Frage angestellt habe, indem jch Lopröse vaccinirte und die Lymphe auf ibren Gehalt an Teprabacillen untersuchte, sind so ausgefallen, wie ich erwartete. Boi verbreiteter cutaner Lepra, wo auch anseheinend normale Haut Bacillen enthält, fanden sich solche anch in der Lymphe, bei reiner Lepra nervorum, wo die Haut frei von Bacillen ist, fehlten sie darin. Sebr bekannt ist der Fall von Gairdner") geworden, wo ein ungenannter Arzt einer ungenannten tropischen Insel soin eigenes Kind von einem später leprös erkrankten eingeboronen Kinde geimpft hat, und ein anderes Kind weisser Eltern wieder von dem seinen. Sowohl der Sohn des Arztes als der von ihm abgeimpfte Kuabe sind später dem Aussatz anheimgefallen. Aus Hawaii kann ich berichten, dass ich einon 12jährigen dentschen Knaben an Lepra behandelt habe, von dem, als or 7 Jalre alt, war, vorschiedene weisse Familien abgoimpft sind. Ich weiss zwar nicht von hierdurch weiter verbreiteter Lepra, doch möchte immerhin ein solches Factum darauf hinweisen, dass man in leprös durchsenchten Ländern eine jegliche Arm zu Arm Vaccination verbieten solle.

In allernenester Zeit hat Ashmead ${ }^{3}$ ) gegen die Hypothese der Uebertragung von Lepra durch die Vaccination vorgebracht, dass, wenn die Krankheit durch Impfung übertragen werden könne, sie in Japan, wo Tä̈towirung so allgemein geübt wird, noch verbreiteter sein müsse, als thatsächlich der Fall ist. Nun

1) Report Hawaiian Board of Health 1886.

3) Brit. Med. Joun. June 11. 1887.

3) Americ. Journ, cut. and gen.-urin. dis: June 1890 p. 220.

Arohiy f: bermatol. a. Syph. 1841. 
ich denke, gegen dieses Argument liesse sich nach verschiedener Richtung hin Einspruch erheben. Den Mosquitostich scheint Ashmead auf der anderen Seite sehr zu fürchten, und ich gebe zu, dass der Gedanke einer Uebertragung der Lepra durch Insectenstiche etwas recht Bestechendes hat. Mich interessirt wesentlich dabei der Umstand, dass die Mosquito-Plage und die Lepra-Plage auf den hawaiischen Inseln eine gewisse zeitliche Uebereinstimmung zeigen. Die früher dort unbekannten Mosquitos wurden Ende der Vierziger Jahre angeblich aus China eingeführt. Ich habe die innerhalb des Bettnetzes schwerer Fälle von cutaner Lepra befindlichen Mosquitos mehrfach bacterioskopisch untersucht. Die prall mit dem Blute des Kranken vollgesogenen Insecten wurden gefangen und einige gleich, einige nach mehrtägiger Gefangenschaft gefärbt und der mikroskopischen Betrachtung unterworfen. Ich konnte keine Leprabacillen in ihnen oder an ihnen nachweisen.

Immerhin ist das wohl ein Punkt, der ebenso wie die Vaccinationsfrage noch weiterem experimentellen Studium zugänglich ist. Auch dürfte es wichtig sein zu erfahren, ob Lepraländer im Allgemeinen Mücken]änder sind. Ein uns bisher unsichtbares Sporenstadium der Bacillen könnte hier, wio bei allen anderen indirecten Uebertragungsmodalitäten, supponirt werden, ohne dass man anf weitergehende Hypothesen von einem Generationswechsel des Leprabacillus in dem Mosquito als Zwischenwirth zu recurriren brauchte.

Unmittelbar mit der Vaccinationsfrage verknüpft, steht die Frage von der Möglichkeit einer beabsichtigten Uebertragung von Lepra von Mensch zu Mensch. Ich habe im vorjährigen ersten Congresse der deutschen dermatologischen Gesellschaft in Prag über meine erfolgreiche Lepra-Impfung an dem Mörder Keanu ausführlichen Bericht erstattet und möchte hier nur kurz das Résumé wiederholen: Ein anscheinend kerngesunder, auffallend kräftiger Mann, der hereditär nicht mit Lepra belastet war, wird Ende September 1884 mit Lepramaterial geimpft, welches unmittelbar einem Kinde entnommen war, das an schwerer tuberöser Lepra litt, und eben ein lepröses Fieber durchgemacht hatte. Die Haupt-Impfung, Finheilung eines Knotenstückchens, geschah am linken Vorderarme. Vier Wochen darauf entstehen rheumatoide 
Schmerzen, zunächst in der linken Schulter, dann auch in anderen Gelenken des linken Aımes und daran sich anschliessend eine schmerzhafte Echwellung des Nervus ulnaris und medianus, Alles ohne Fieber und mit ausserordentich geringer Störung des Allgemeinbefindens.

Im Laufe der nächsten 6 Monate geht die Nowritis allmälig zurück und es kommt zur Bildung eines kleinen Lepraknotens an der keloidartig verheilten Impfstelle. Im Gewebssafte dieses Narbenkeloids lassen sich 16 Monate nach der Impfung noch Leprabacillen nachweisen. Im September 1887 werden schon deutliche Symptome von Lepra beobachtet und 1 Jahr darauf finden wir den Patienten bereits auf der Höho der Erkrankung.

Anamnestisch hatte ich hervorgehoben, dass, soweit ich damals von Keanu selber und von Solchen, die ihn kannten, erfabren konnte, seine Familie für hawaiische Verhältnisse merkwürdig froi von Lepra war, dass aber diese Anamnese wie alle derartigen Erhebungen bei Hawaiiern vur cum grano salis zu nehmen sei. Schon jetzt hat sich gezeigt, wie richtig diese Reserve war. Der Arzt der Lepra-Colonie Molokai, Dr. Swift, hat jotzt einen Neffeu und den Sohn Keanu's als Leprafïlle unter sich, auch ein Vetter Keanu's hat sich als leprös herausgestellt. Diesen Thatsachen entgegen muss jeh aber wiederum hervorheben, dass Keauu selber, jedenfalls zur Zeit der Impfung, Lnde September 1884, frei von der Krankheit war, dass er hingegen im Septembor 1887 bereits deutliche Zeichen der Erkrankung anfwies, und jetzt einen typischen Fall von allgemeiner Lepra darstellt. Dr. Swift meint nun, dass es ibm kaum glaubhaft erscheint, dass die Krankheit in 5 Jahren zu so sehweren Symptomen führen könne. Dem gegenüber will ich nur einen gleichfalls sehr bekannten Fall von Lepra erwähnen, der einen noch rapideren Verlauf genommen hat, den des allbekannten Pater Damien. Im Juli 1882 erkrankte Damien an einer Neuralgie des linken Fusses, am 25. November 1884 untersuchte ich ihn, fand eine typische Analgesie und Hantatrophie an der Aussenseite des linken Fusses mit einer Schwellung des Nervus peroneus superficialis, und machte ihm kein $\mathrm{Hehl}$ aus meiner Diagnose Lepra. $1886 \mathrm{kam}$ es zu den ersten Infiltrationen an der Gesichtshaut und im Juli 1889 war der bedauernswerthe Mann seiner Krankheit bereits erlegen. 
Da haben wir einen Ietalen Verlauf von den allerersten Erscheinungen an in 7 Jahren, vom Beginne des tuberösen Stadiums an in 3 Jahren. Wenn auch die Beispiele einer so rasch verl aufenden Lepra vicht gerade häufig sind, so könnte ich doch noch eine ganze Reihe derselben aus meinen hawaiischen Erfahrungen anführen.

Bei Beurtheilung der Tragweite meines Inoculationsversuches möchte ich bitten, sich nicht an die nackte Thatsache zu halten, dass der mit Lepra Inoculirte nach 3 Jahren schon eine ausgesprochene Lepra zeigte, sondern das Detail des Versuches zu beachten. Um einen positiven Beweis zu bringen, muss die bacteriologische Lepraforschung ihr Hauptbestreben darauf richten, Kriterien des Lebens der Leprabacillen zu finden. Die Klippe, an der bisher unsere Untersuchungen scheitern, ist entschieden die, dass wir nicht anzugeben vermögen, ob wir in dem jeweiligen Falle lebende oder todte Bacillen ror uns haben. Wir wären einen grossen Schritt weiter, wenn wir auf irgend eine Weise nachweisen könnten, ob zum Beispiel die mit den Fäces bei Darmlepra ausgeschiedenen Bacillen lebensfähig sind oder nicht, ob die bei der Leichenfäule und jahrelanger Maceration von Lepragewebe persistirenden Bacillen lebende Infectionsträger darstellen oder nicht. Hätten wir ein Kriterium des Lebons der Leprabacillen, wie wir es zum Beispiel für die Tuberkelbacillen haben, so hätte ich den Beweis beibringen können, dass die 14 Monate nach der Inoculation bei dem Manne Keanu an der Impfstelle gefundenen Leprabacillen lebend waren, während nach dem heutigen Stande unserer Kenntniss ich mir den Einwand gefallen lassen muss, dass es wie ein todter Fremdkörper eingeheilte Bacillen des übertragenen Gewebestückchens gewesen sind.

Erst, wenn es der Bacteriologie gelungen sein wird, durch den Fund bestimmter mikroskopischer und biologischer Eigenschaften, durch Culturverfahren oder stets sicher gelingende Thierimpfung eine greifbare Differenz zwischen lebenden und todten Leprabacillen nachzuweisen, kann die Frage über die Infectionsmodalitäten, über directe Contag ion oder Infection durch Vermittelung von Boden, Wasser oder Nahrung ihrer definitiven Lösung entgegengebracht werden. 